\title{
MICROMACHINED SCANNING REFRACTIVE LENS
}

\author{
Daniel A. Fletcher, Kenneth B. Crozier, Gordon S. Kino, Calvin F. Quate, and Kenneth E. Goodson \\ E. L. Ginzton Laboratory, Stanford University \\ Stanford, CA 94305
}

\begin{abstract}
A refractive lens placed close to a surface can be used to obtain resolution below the optical diffraction limit in air. The improvement in resolution is proportional to the index of refraction of the lens, called a Solid Immersion Lens (SIL). Small lenses (diameter $\sim 10 \mu \mathrm{m}$ ) made with micromachining techniques offer several advantages over large SILs (diameter $\sim 1 \mathrm{~mm}$ ) including reduced aberrations and, when integrated onto cantilevers, control of lens-sample separation. We report here the fabrication and demonstration of the first micromachined scanning refractive lens. The integrated lens and cantilever is made from single-crystal $\mathrm{Si}$ and operated in transmission mode.
\end{abstract}

\section{INTRODUCTION}

Scanning probes such as the STM and AFM characterize surfaces through local electric, magnetic, and other interactions. Optical imaging below the diffraction limit can be obtained by scanning a sub-wavelength source or collector such as a tapered optical fiber above the surface, a technique known as scanning near-field optical microscopy (SNOM) [1]. Sub-wavelength sources can be created by forcing light through small apertures like those on tapered fibers. This approach suffers from low optical efficiency and difficulty manufacturing reliable apertures. An alternate and more efficient technique for high-resolution imaging is solid immersion microscopy, which uses the index of refraction of a lens held against the surface to reduce spot size [2].

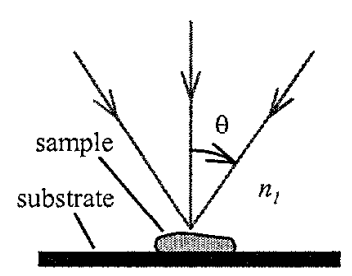

$\mathrm{NA}=n_{l} \sin \theta$

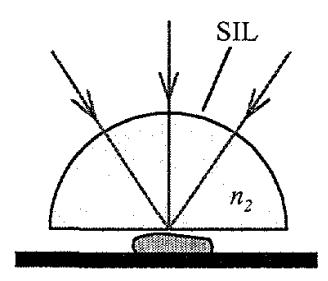

$\mathrm{NA}=n_{2} \sin \theta$
Figure 1. Focussing through air and through a hemispherical SIL. The minimum resolvable feature size is improved by $n_{2} / n_{1}$ with use of the SIr

The spatial resolution of a lens without aberration is limited by diffraction of light from the lens aperture. The minimum fullwidth half-maximum (FWHM) resolution, $w$, in the scalar approximation is $w=\lambda / 2 \mathrm{NA}$, where $\lambda$ is the free space wavelength, $\mathrm{NA}=n \sin \theta$ is the numerical aperture, $\theta$ is the maximum angle of incidence, and $n$ is the index of refraction at the focus. Figure 1 shows light focused on a sample through a medium of index $n_{l}$ and through a hemispherical SIL of index $n_{2}$ and radius $a$ onto the same sample. In the case of imaging in air, $n_{1}=1$, the improvement in resolution through the hemispherical SIL is $n_{2} / n_{l}=n_{2}=n$. If the lens is a supersphere, where the light is focussed to a point $a / n$ from the center of the spherical surface, refraction at the lens further increases resolution for angles in air below the maximum $\theta_{m}$ given by $\sin \theta_{m}=1 / n$. The highest resolution for both hemisphere and supershpere SILs is the same and occurs when $\sin \theta$ in the lens is 1.0 .

Terris et al. [3] were able to write $317-\mathrm{nm}$ bits in a magnetooptic material with a $n=1.83 \mathrm{SIL}$ at $\lambda=780 \mathrm{~nm}$. Ghislain et al. [4] exposed 190-nm lines in photoresist using a $n=2.2$ SIL at $\lambda=$ $442 \mathrm{~nm}$. Other work has used SILs for high-resolution microscopy and phase-change data storage [5-6]. Further improvernents in resolution may be possible with short wavelengths and high-index materials, such as $\mathrm{Si}_{3} \mathrm{~N}_{4}(n=2.0), \mathrm{SrTiO}_{3}(n=2.4)$, or $\mathrm{Si}(n=3.4)$.

The sample surface must be positioned within the near field of the SIL for optimum resolution, approximately $100 \mathrm{~nm}$ for visible light. If topographical variations in the sample cause a greater separation between lens and sample, resolution is reduced because of the exponential fall-off of the focussed fields in air [7]. Lenses can be made with tips on the order of $1 \mu \mathrm{m}$ in diameter to localize contact between the SIL and sample, and images can be constructed by scanning the lens across the sample as with the AFM. Integrating a small lens onto an AFM cantilever allows scanning across high spatial-frequency topography with force feedback control. Traditional methods for manufacturing SILs cannot be used to make lenses smaller than approximately $1 \mathrm{~mm}$ in diameter. Micromachining offers a relatively inexpensive and repeatable method for batch fabrication of integrated lenses and cantilevers.

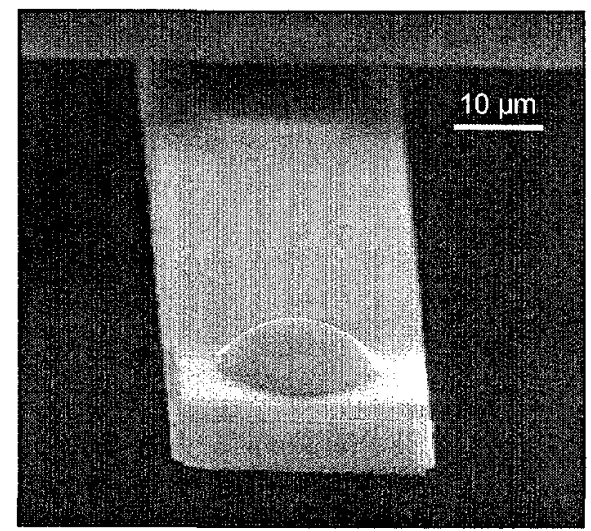

Figure 2. Integrated refractive microlens and cantilever fabricated from single-crystal Si.

Micromachined lenses also benefit from reduced aberrations and sensitivity to focal position in the propogation direction. As lens diameter is reduced, spherical aberration is reduced, and lenses smaller than $10 \mu \mathrm{m}$ have been shown through an extension of Mie theory [8] to focus both plane waves and converging waves of $\lambda=0.633 \mu \mathrm{m}$ to nearly the same spot size [9]. Lenses with diameters as small as one wavelength have also been shown to 

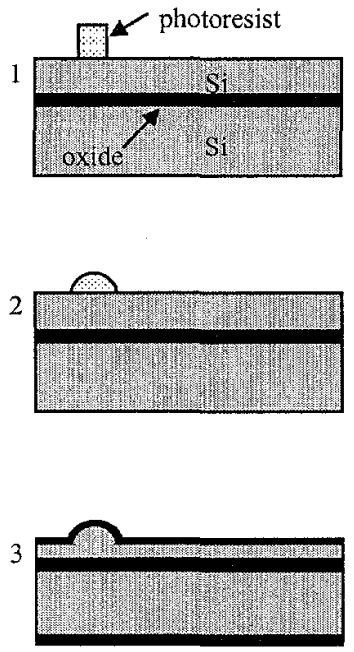
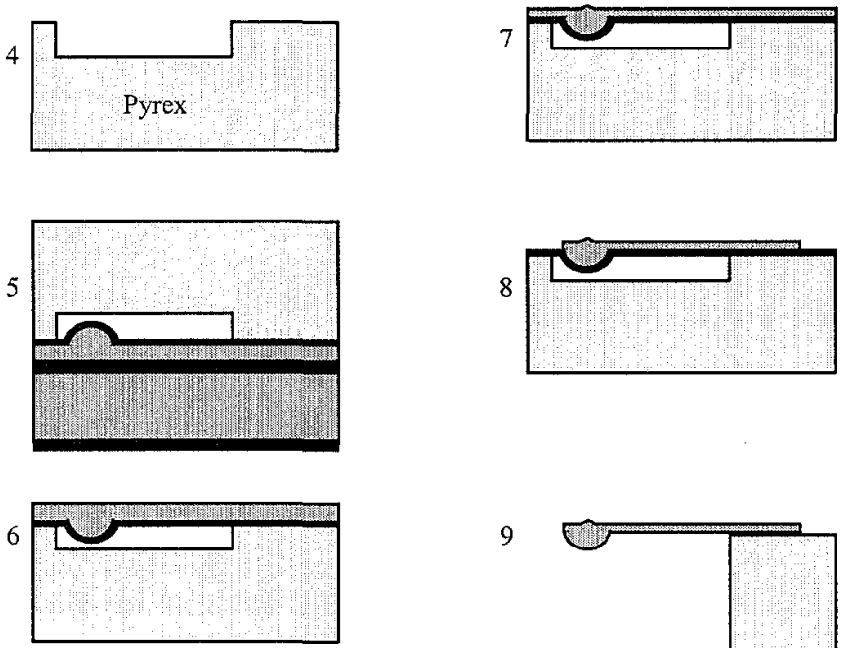

Figure 3. Process flow for fabrication of the integrated cantilever and lens. (1) Pattern photoresist pillars. (2) Reflow pillars in acetone vapor. (3). Transfer shape into upper Si and grow thermal oxide. (4) Etch pits in Pyrex. (5) Anodically bond Pyrex and upper Si. (6) Etch substrate Si in TMAH and clear middle oxide in BOE. (7) Pattern tip with photoresist and isotropically dry etch. (8) Pattern cantilever with photoresist and anisotropically dry etch. (9) Release with wafer saw.

focus light, though with a field distribution that differs from vector diffraction theory.

\section{MICROLENS FABRICATION}

Cantilevers with lenses on the order of $10 \mu \mathrm{m}$ in diameter are fabricated from the upper single-crystal Si of a silicon-on-insulator (SOI) wafer. An integrated microlens and cantilever is shown in Fig. 2. The fabrication process begins by defining a pillar in photoresist, as shown in Fig. 3. The pillar is reflowed in acetone yapor to achieve the desired lens shape. The shape is a function of reflow time, acetone vapor concentration in the sealed container, and aspect ratio of the original photoresist pillar. After baking at $110^{\circ} \mathrm{C}$ for 20 minutes to drive off solvents, the shape is transferred into the upper $\mathrm{Si}$ with a non-selective $\mathrm{CF}_{4} / \mathrm{O}_{2}$ reactive ion etch [10]. The shape of the lens can be further adjusted during the transfer etch by varying the relative Si:photoresist etch rate. For example, a hemispherical shape transferred with an etch preferential to photoresist will result in a flattened Si lens shape. A $0.3-\mu \mathrm{m}$ thick thermal oxide layer is grown on the lens to reduce roughness and serve as an etch stop for the cantilever etch.

A tip is needed opposite the lens at the focal point to localize the contact between lens and sample for scanning. To access the lower face of the upper Si layer, a Pyrex handle wafer is anodically bonded to the top Si at $350^{\circ} \mathrm{C}$. The Pyrex wafer is patterned with pits and etched in a $\mathrm{HF} / \mathrm{HNO}_{3} / \mathrm{H}_{2} \mathrm{O}$ mixture. The pits protect the lenses and define cantilever length. The bulk substrate $\mathrm{Si}$ is removed with a $25 \% \mathrm{TMAH}$ etch, and the middle oxide is cleared with $6: 1$ BOE. Tips are then patterned on the suspended Si membrane and etched in an isotropic reactive ion etch. The cantilevers are patterned and isotropically etched, stopping on the thermal oxide layer grown earlier. The oxide is removed in 6:1 BOE to release the cantilevers. Finally, chips with integrated lenses and cantilevers are cut by wafer saw. A one-dimensional array of integrated lenses and cantilevers is shown in Fig. 4.

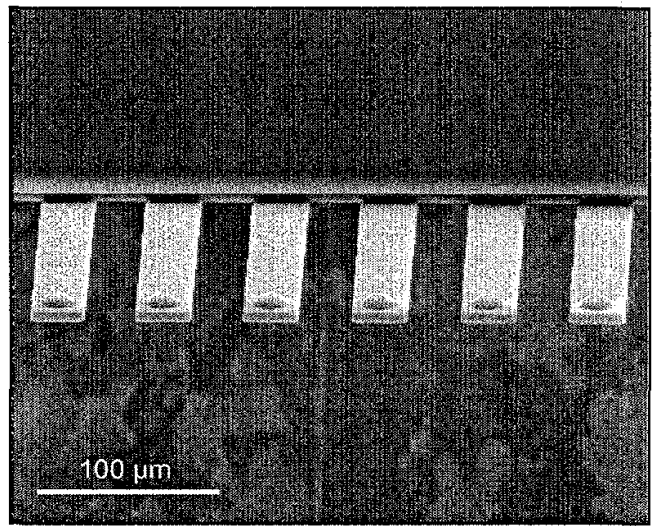

Figure 4. One-dimensional array of integrated refractive microlenses and cantilevers.

\section{EXPERIMENTS}

We have used the micromachined scanning lens to focus $0.633-\mu \mathrm{m}$ light and to collect a two-dimensional image of a $0.2 \mu \mathrm{m}$ line-width grating in the transmission mode. Focussing was demonstrated by imaging a laser spot with and without the micromachined lens. Figure $5 \mathrm{~A}$ shows a HeNe laser spot $(\lambda=$ $0.633 \mu \mathrm{m})$ created by a low-angle objective ( $\sin \theta=0.04)$ and imaged with a $C C D$ camera. When the micromachined lens is inserted near the focal point, as shown in Fig. 5B, the spot size is reduced by a factor of 15.7 .

The improvement in spot size is due to the index of refraction of Si $(n=3.8$ at $\lambda=0.633 \mu \mathrm{m})$ and refraction at the lens surface. Refraction occurs because the SIL is operating like a supersphere, in which the tip is below the center of the sphere and light focussed on the tip enters at an angle to the radius of the sphere. The maximum angle of incidence increases such that $\sin \theta$ is a 

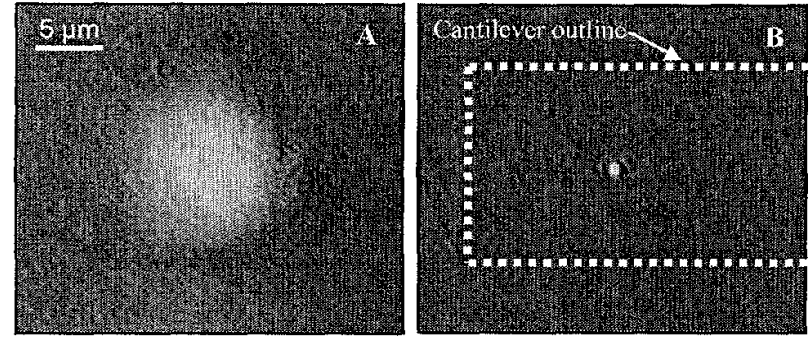

Figure 5. Focussing by the microlens. (A) HeNe laser spot $(\lambda=$ $0.633 \mu \mathrm{m})$ created by a low-NA objective $(N A=0.04)$. (B) HeNe laser spot focussed through the micromachined Si lens.

factor of 4.0 greater with the lens than without. A comparison of the spots in Fig. 5 fit to a Guassisan profile is presented in Fig. 6 .

The RMS surface roughness of the lens is estimated from AFM scans to be less than $2 \mathrm{~nm}$. Total integrated scattering due to the roughness is less than $0.5 \%$. Note that bulk Si absorbs at $\lambda=$ $0.633 \mu \mathrm{m}$, but the lens is partially transparent. If micromachined lenses are made thin enough, it is possible to use absorbing, highindex materials such as $\mathrm{Si}$ and achieve good transmission efficiency.

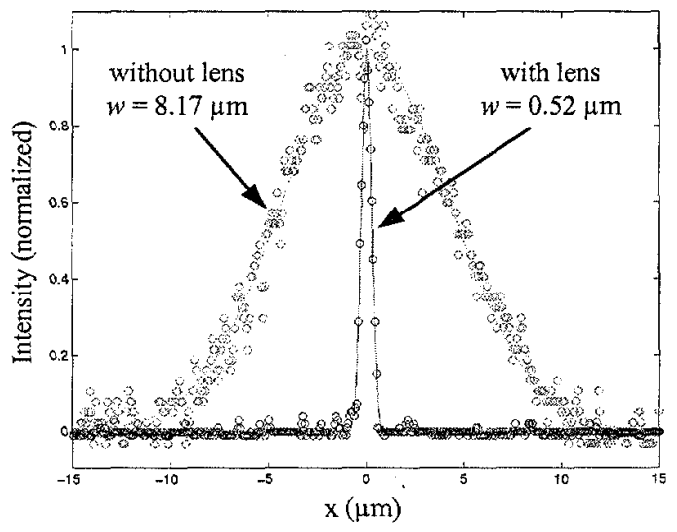

Figure 6. Comparison of the FWHM size, w, of the focussed spots shown in Fig. 5 fit to a Guassian profile.

Conventional lenses form images of adjacent points within their field of view. In contrast, a micromachined lens integrated onto a scanning probe with a tip to localize contact forms an optical image serially in a manner similar to an AFM. We have demonstrated scanned image formation by moving an amplitude grating beneath a single lens in contact mode and collecting the transmitted light. The grating is $0.1-\mu \mathrm{m}$ thick Ti on quartz with a line-width of $0.2 \mu \mathrm{m}$, and the cantilever was operated without force feedback. A HeNe laser $(\lambda=0.633 \mu \mathrm{m})$ was chopped and focused onto the micromachined lens with a high-angle objective ( $\sin \theta=0.8$ ). Transmitted light was collected with a second highangle objective ( $\sin \theta=0.8$ ) and measured with a photodetector and lock-in amplifier. A two-dimensional scan of the $0.2-\mu \mathrm{m}$ linewidth grating is shown in Fig. 7, with the normalized transmitted intensity ranging from 0.35 over a line to 0.65 over a space.

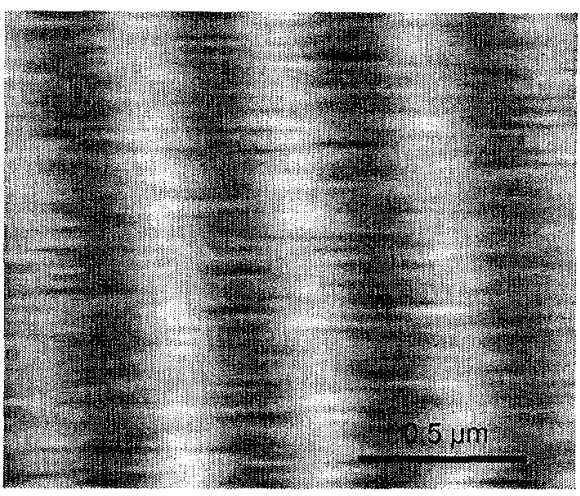

Figure 7. Two-dimensional image of a $0.2 \mu m$ line-width Ti-onquartz grating taken in transmission mode by scanning the grating beneath a HeNe laser focussed through the micromachined Si SIL.

The resolution of the image taken with the SIL was estimated from the amplitude variation to be a factor of 2.8 greater than the same image taken without the SIL. When a high-angle objective is used to illuminate the SIL, refraction at the lens surface does not contribute significantly to a reduction in spot size. Aberrations in the incident beam or offsets in alignment may account differences from the maximum factor of 3.8 improvement from index of refraction. The minimum feature size that can be resolved with this technique is currently being investigated. Recent experiments at $\lambda=9.2 \mu \mathrm{m}$ indicate that resolution as high as $\lambda / 6$ can be achieved with the Si SIL.

\section{CONCLUSIONS}

Solid Immersion Lenses micromachined from high-index materials will improve optical resolution in applications such as imaging and lithography. Micromachining offers a repeatable method for batch fabrication, and small lenses have reduced spherical aberration and improved tolerance to lens shape and wavefront errors. We have demonstrated the fabrication and operation of micromachined refractive lenses on the order of 10 $\mu \mathrm{m}$ in diameter. The lenses are integrated onto a cantilever for scanning and made with a tip to localize lens-sample contact. Results indicate that improvements in minimum resolution by a factor of almost 3 can be achieved with Si lenses.

\section{ACKNOWLEDGEMENTS}

The authors thank Daniel Palanker and Dmitri Simanovski for their collaboration on the infrared measurements. This work was conducted with support from the Department of Energy under contract DEFG03-90ER14157 and SRC Contract 754. We made use of the Stanford Nanofabrication Facility, part of the National Nanofabrication Users Network funded by the National Science Foundation under award ECS-9731294. DAF acknowledges support of the NSF through a Graduate Research Fellowship, and KEG acknowledges support from the NSF Career Award.

\section{REFERENCES}

1. E. Betzig and J. K. Trautman, "Near-Field Optics: Microscopy, Spectroscopy, and Surface Modification Beyond the Diffraction Limit", Science, 257, 189 (1992). 
2. S. M. Mansfield and G. S. Kino, "Solid Immersion Microscope", Applied Physics Letters, 57 (24), 2615 (1990).

3. B. D. Terris, H. J. Mamin, D. Rugar, W. R. Studenmund, and G. S. Kino, "Near-field optical data storage using a solid immcrsion lcns," Applied Physics Letters, 65 (4), 388 (1994).

4. L. P. Ghislain, V. B. Elings, K. B. Crozier, S. R. Manalis, S. C. Minne, K. Wilder, G. S. Kino, and C. F. Quate, "Near-field photolithography with a solid immersion lens," Applied Physics Letters, 74 (4), 501 (1999).

5. Q. Wu, G. D. Feke, R. D. Grober, and L. P. Ghislain, "Realization of numerical aperture 2.0 using a gallium phosphide solid immersion lens," Applied Physics letters, 75 (26), 4064 (1999).

6. K. Kishima, I. Ichimura, K. Yamamoto, K. Osato, Y. Kuroda, and K. Saito, "Near-Field Phase-Change Optical Recording over 1.2-Numerical-Aperture," Proceedings of the 1999 International Symposium on Optical Memory and Optical Data Storage, Hawaii, 7/11-15/1999, SPIE (1999).

7. G. S. Kino, D. A. Fletcher, K. B. Crozier, K. E. Goodson, and C. F. Quate, "An Efficient Near-field Microscope for Thermal Measurements," Proceedings of the Seventeenth Symposium on Energy Engineering Sciences, Argonne National Labs., 5/1314/99, Department of Engergy (1999).

8. M. Born and E. Wolf, Principles of Optics, $6^{\text {th }}$ edition, Cambridge: Cambridge University Press, 1980.

9. D. A. Fletcher, K. B. Crozier, K. E. Goodson, C. F. Quate, and G. S. Kino, "Optical characterization of microfabricated solid immersion lenses," Proceedings of the 2000 International Symposium on Biomedical Optics, Photonics West, San Jose, CA 1/22-28/2000, The International Society for Optical Engineering (SPIE), Bellingham, WA (2000).

10. D. A. Fletcher, K. B. Crozier, G. S. Kino, C. F. Quate, and K. E. Goodson, "Fabrication of High-Index Refractive Microlenses for Near-Field Optics", Proceedings of the 1999 ASME International Mechanical Engineering Congress and Exposition, MEMS Symposium, Nashville, TN, 11/14-19/99, American Society of Mechanical Engineers, New York (1999). 